\title{
Intelligent Wakening Scheme for Wireless Sensor Networks Surveillance
}

\author{
Rui Wang, Lei Zhang, Li Cui \\ Institute of Computing Technology of the Chinese Academy of Sciences, Beijing, China 100190 \\ \{wangrui,zhanglei82,lcui\}@ict.ac.cn
}

\begin{abstract}
The effective energy control while maintaining reliable detection performance is a key problem in wireless sensor networks surveillance, such as blue-green algae surveillance. An intelligent wakening scheme (IWS) is proposed in this paper which considers different importance degrees of the grids in the surveillance zone. It uses a voronoi diagram to determine the effective scope of each sensor node and calculates the node wakening probability with the importance degree in the effective scope. The nodes are then turned on stochastically according to the node wakening probability. Simulation results illustrate that this scheme greatly reduces the number of wakening nodes while maintaining high reliability in surveillance.
\end{abstract}

Keywords-wireless sensor networks; intelligent wakening scheme; energy control; blue-green algae surveillance

\section{INTRODUCTION}

The maturing of integrated circuitry, digital signal processing, micro electromechanical systems (MEMS) and low-range radio electronics on a signal node have led to the design of wireless sensor networks (WSN), which have attracted many researchers due to its wide range application potentials. A WSN provides a new class of computer system and expands human ability to remotely interact with the physical world. Applications of WSN include battlefield surveillance, biological detection, home appliance, smart spaces and inventory tracking. By applying WSN in the monitoring of Blue-green Algae Bloom on Lake Tai[1], we can obtain the physical information, like water temperature and water color, and it is possible to predict biological growth and potential ecological disaster.

WSN has some unique characteristics, of which the most importance one is limited energy supply. A sensor node has a finite energy reserve supplied by a battery. It is often unfeasible to recharge the node's battery. Thus minimizing energy consumption while maximizing the system lifetime is a major objective in designing wireless sensor networks.

Generally, the energy consumption of sensor nodes consists of three parts: the microprocessor, the radio communication module and the senor module. When the sensor node is put into working status all the time, sensor module is often switched on and the radio module is turned on when necessary. Although the radio communication is the largest energy consumer, we can also save much energy by reduce the working time of sensor module.
In wireless sensor networks, adjacent nodes share common sensing tasks, which implies that not all sensors are required to perform the sensing task during the whole system lifetime. That is to say, the function of whole system will not be affected by some sleeping nodes as long as there are enough working nodes. Therefore, if the sensors can be well scheduled, the system lifetime can be prolonged correspondingly; i.e. the system lifetime is prolonged by exploiting redundancy.

In this work, IWS (Intelligent Wakening Scheme) is presented. In IWS, every node has a local decision on whether it needs to be turned on or off by dynamic calculation of its importance degree in WSN. Our design has been driven by the following requirements: firstly, the self configuration is mandated because it is inconvenient or impossible to manually configure sensors after they have been deployed in hostile or remote working environments. Secondly, the design has to be fully distributed and localized. Finally, the algorithm should provide a differentiated surveillance service for different importance degree areas while maintaining high enough detection probability to targets.

\section{RELATED WORKS}

Meguerdichian et al. [2] addressed one of the fundamental problems, namely, coverage, which in general answers the questions about the quality of service that can be provided by a particular sensor network.

A node-scheduling scheme by [3] turns some nodes on or off while guarantees certain redundancy. A node will turn off if it discovers that its neighbors can substitute it to monitor its sensing area. The solution does not need the global knowledge of the network and thus can be implemented locally at each node. The proposed scheme increases communication cost, requires synchronization and involves in the calculation of a geometric representation's intersection. In [4], Ye et al. developed a distributed density control algorithm named PEAS, which is probing based. In PEAS, each node sleeps for an exponentially distributed duration. A sleeping node wakes up and broadcasts a probing message within a certain range after its sleeping period; if no reply is received after a timeout, it will turn on to work until it depletes its energy.

Previous research [2-7] focused on how to provide full or partial sensing coverage in the cost of energy consumption. In such an approach, a node will be set to be asleep as long as its neighbors can cover its sensing area. These solutions treat the 
different surveillance zones with the same importance. However, in most scenarios such as battlefields, there exist some geographic sections such as the general command center that is much more security-sensitive. Based on the fact that individual sensor nodes are not reliable and subject to failure and single sensing readings can be easily distorted by background noise and cause false alarms, it is simply not sufficient to rely on a single sensor to safeguard a critical area. In this case, it is desired to provide higher degree of coverage in which multiple sensors monitor the same location at the same time in order to obtain high confidence in detection. However, it will be too energy consuming if the same high degrees of coverage are applied in some non-critical areas.

The rest of the paper is organized as follows: The details of IWS algorithm are discussed in Section 3. Simulation results are presented and discussed in Section 4. Concluding remarks are provided in Section 5.

\section{INTELLIGENT WAKENING SCHEME}

In this paper, "Importance Degree" and "Effective Scope" are introduced into IWS. By this way, the importance of individual sensing scope is quantified. The waken up probability is decided by the quantified value. More important degree will be assigned a larger wake up probability. In this way, we reduce the number of working nodes while keeps Surveillance performance and save more energy.

\section{A. Importance Degree}

According to the experience of previous blue-green algae surveillance, the green algae outbreak possibility differs in different water areas, it is influenced by water temperature weather conditions even geological terrain. Correspondingly, water area which has a high possibility of green algae outbreak, should be allocated more sensing resource to assure the timely outbreak prediction.

In our design, the surveillance zone is divided into a lot of discrete grids. Define the importance degree of a grid as the frequency of targets appearing in the grid. The similar idea "Certainty Grids" has been used successfully in several mobile robot control programs [8]. It can be obtained through a priori information. The different importance of the grids in the surveillance zone can be directly displayed by their different importance degrees. The higher the importance degree is, the higher the frequency targets appear, and the more importance the grid is.

Consider the case where the surveillance zone D is a 2Dimension region, then it can be divided into $m \times n$ grids $D=\left\{g_{i j}\right\}_{m \times n}$ and an importance degree matrix is defined as follows, $A=\left[a_{i j}\right]_{m \times n}$, with $a_{i j}$ representing the importance degree of grid $g_{i j}$.

In this paper, each grid is be sensed with an exponentially distributed duration generated according to a probability density function $f^{i j}(t)=a_{i j} e^{-a_{i j} t}$, where $a_{i j}$ is the importance degree of the grid, $t$ denotes the time duration and is set by mission requirements. The more important the grid is, the higher frequency it should be sensed.

Obviously, $E^{i j}\{t\}=1 / a_{i j}$ is actually the mean of intervals to be sensed. Then the probability distribution function of grid $g_{i j}$ at time $t$ is as followed:

$$
F_{x}^{i j}(t)=P(x \leq t)=1-e^{-a_{i j} t}
$$

At time $t$, the larger $a_{i j}$ is, the larger the value of $F_{x}^{i j}(t)$ is.

\section{B. Effective Scope of Node}

In this paper, the Voronoi diagram is used to define the effective scope of the nodes.

Let $S=\left\{p_{1}, p_{2}, \ldots, p_{n}\right\}$ be an aggregation of points in a two-dimensional Euclidean plane. These points are called sites. A Voronoi diagram decomposes the space into regions around each site, so that the points in the grids around $p_{i}$ are closer to $p_{i}$ than any other point in $\mathrm{S}$.

Using the definition in [9], the Voronoi region $V\left(p_{i}\right)$ for each $p_{i}$ is expressed as:

$$
V\left(p_{i}\right)=\left\{x: d\left(p_{i}, x\right) \leq d\left(p_{j}, x\right), \forall j \neq i\right\}
$$

$V\left(p_{i}\right)$ consists of all points that are closer to $p_{i}$ than any other site. The aggregation of all sites form the Voronoi Diagram $V(s)$.

If there are denser nodes in surveillance zone, the voronoi region acreage will be smaller because of large overlapped proportion.

\section{The Algorithm}

Here we will propose the IWS (Importance Degree based Wakening Scheme) that calculates node wakening probability according to the different importance degrees of surveillance grids. To simplify the problem, we assume that all nodes have the same sensing range $\mathrm{r}$ and communication range $R_{c}$.

We consider the S-MAC protocol [10] for the wireless sensor networks communication, where in each operation period, also called Round, the nodes operate in two phases. In the first phase, the node determines whether it should be awake or not stochastically according to the wakening probability. If it chooses wakening state, it will implement detection and may further do some simple computation. Otherwise it will just turn off so that the energy can be saved. In the second phase, the node communicates with its neighbors through receiving or sending information. The operation flow can be represented as Fig.1.

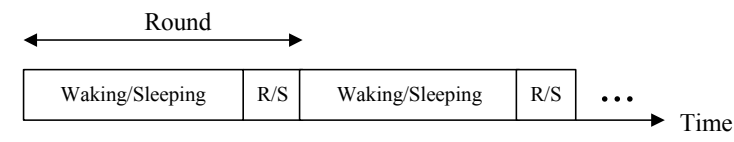

Figure 1. Operation flow of ant nodes operation.

The sensor detection model is considered as followed: 


$$
C_{x}(i)= \begin{cases}1 & \{x: d(x, i) \leq r, \forall x \in R\} \\ 0 & \{x: d(x, i)>r, \forall x \in R\}\end{cases}
$$

Where $\mathrm{R}$ is the zone of surveillance, $d(x, i)$ denotes the distance between node $i$ and geographical location point $x$.

Given sensor nodes $S=\left\{p_{1}, p_{2}, \ldots, p_{n}\right\}$, the Voronoi Diagram is: $V(s)=\left\{V\left(p_{1}\right), V\left(p_{2}\right), \ldots, V\left(p_{n}\right)\right\}$ where $V\left(p_{i}\right)$ is the Voronoi region satisfying (2). Then, we define a mapping from $V\left(p_{i}\right)$ to $U\left(p_{i}\right)$, as followed:

$$
U\left(p_{i}\right)=\left\{g_{i j}: g_{i j} \in V\left(p_{i}\right)\right\}
$$

Define the weight of the Voronoi region $V\left(p_{i}\right)$ at time $t$ $I\left(p_{i}, t\right)$ as the sum of $F_{x}^{i j}(t)$ in the region. Given $F_{x}^{i j}(t)$ and $U\left(p_{i}\right), I\left(p_{i}, t\right)$ can be calculated as follows:

$$
I\left(p_{i}, t\right)=\sum_{g_{i j} \in U\left(p_{i}\right)} F_{x}^{i j}(t)
$$

$I\left(p_{i}, t\right)$ explicates the total amount of probability of the grids to be sensed. As we can deduce from (5) that numerous grids and high probability $F_{x}$ result in the large weight $I$. Obviously, the large $I\left(p_{i}, t\right)$ may indicate an urgent detection task on the node $p_{i}$. Then the node wakening probability may be calculated from $I\left(p_{i}, t\right)$. In this paper, we construct the calculation as follows:

$$
W(i, t)=\min \left\{C \cdot \frac{I\left(p_{i}, t\right) \cdot N}{M}, 1\right\}
$$

Where $N$ is the amount of sensor nodes in the surveillance zone, $C$ is the parameter chosen by experimentation, and $M$ is the total amount of grids contained in surveillance zone D that can be calculated as follows:

$$
\mathrm{M}=|D|
$$

After the node wakening probability $W(i, t)$ is calculated, the sensor node can make a stochastic decision on switching from sleeping to activity accordingly.

If a target is detected by an active node $i$, the nodes around it will be aroused. Otherwise they will return to sleep and set $t$ to 0 .

\section{EXPERIMENTAL ANALYSIS}

In this section, we present detailed simulation results to verify the effectiveness of our algorithm. To evaluate the algorithm performance and energy saving result, and to evaluate the parameter choice of " $\mathrm{C}$ " in algorithm performance, we use algorithm with fixed wakening probability as baseline algorithm. We also adopt different value of " $\mathrm{C}$ " to conduct the simulation.

\section{A. Simulation Environment}

Suppose that the blue-green algae surveillance range is $100 \times 100$, and is divided into a lot of discrete grids with $2.5 \times 2.5$. There are 100 sensor nodes randomly scattered in the zone, and the location information is available to the sensor node either through hardware such as embedded GPS or through location discovery algorithms [11]. Furthermore, we assume that the radio communication radius of sensor node always meets the critical density[12] conditions, which means the network is connected all the time. Hence the desired area to monitor of a sensor node is the polygon that is defined by the Voronoi diagram. As figure 2 shows, the nodes do not distribute evenly due to random configuration in real applications.

Here the sensing range $r$ in (3) is set to be 20 .

Suppose that before WSN deployment, we have assigned proper importance degrees to grids according to temperature, weather conditions and geological terrain (High outbreak possibility will be assigned high important degree) .

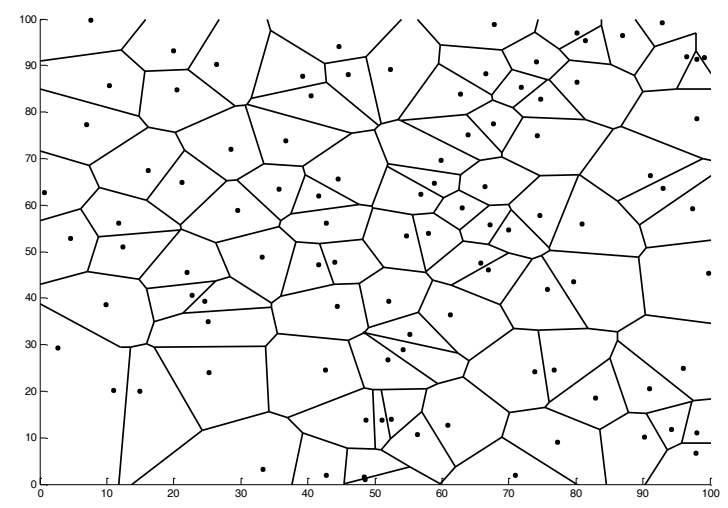

Figure 2. The sensor nodes and its Voronoi diagram

The different importance degrees in the surveillance zone are showed in fig 3. Define the whole surveillance zone as D, where the importance location is figured as the green pentagram named G. G may be a road or a battlefield etc. The sensing mission requires the grids in $\mathrm{G}$ to be detected in $t_{1}=2.5$ second interval while the other grids to be detected in $t_{2}=5$ second interval. Then $a_{i j}$ can be calculated as follows:

$$
a_{i j}=\left\{\begin{array}{rr}
1 / t_{1} & g_{i j} \in G \\
1 / t_{2} & g_{i j} \in D-G
\end{array}\right.
$$

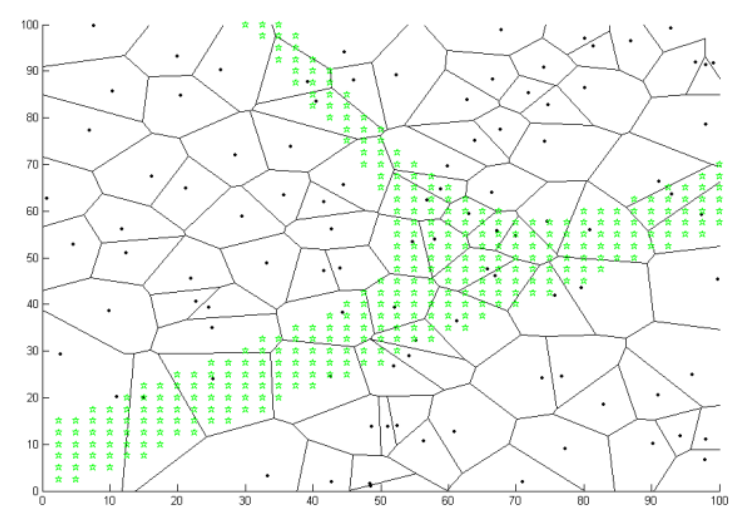

Figure 3. The importance location figured as the area with green pentagram 
Given sensor node $i$ and grid unit $g_{i j}$, the corresponding grids set $U\left(p_{i}\right)$ can be calculated according to (4). $F_{x}^{i j}(t)$ and $I\left(p_{i}, t\right)$ can be calculated according to (1) and (5) respectively. At last, the nodes wakening probability can be calculated according to (6) and (7).

In order to evaluate IWS in a dense targets environment, we generate the targets (green algae outbreak event) stochastically with the number $K=\{50,100,150,200\}$ respectively. And the probability targets appearing in $\mathrm{G}$ (the importance location) is twice of that in the other location. Fig 4 is a snapshot of 200 targets generated in our simulation.

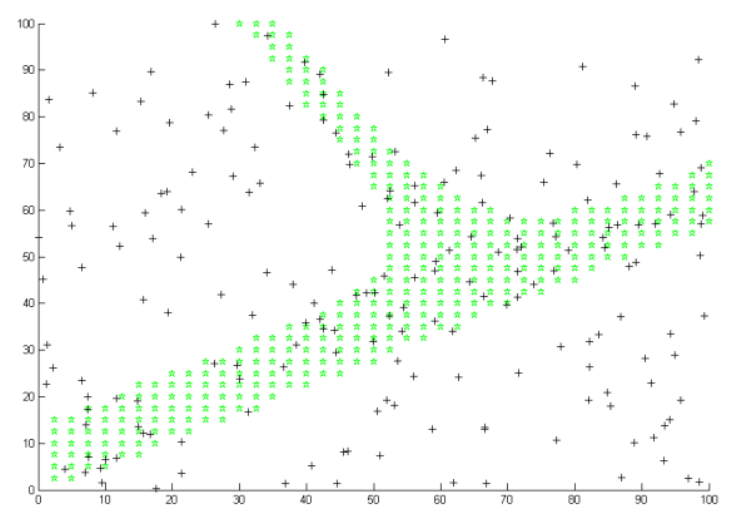

Figure 4. Targets appearing in the surveillance zone

\section{B. Results Analysis}

Scenario. Before each simulation, we initialize the system by setting the time $T_{0}=0$. Every grid is set to activity according to the calculated node wakening probability. At time $T_{0}+1, K$ random targets occur in the surveillance area. When all the targets are detected the simulation is demonstrated successful and the simulation time $T$ is recorded. If there are still targets fail to be detected by the time $T_{0}+5$, then the simulation is demonstrated a failure.

Evaluation. In most wireless sensor networks, energy is a key performance criterion. In our experiment, energy $E$ is defined as the accumulated times of wakening nodes during one simulation. The smaller $E$ is, the less energy the system consumes. Simulation time $T$ demonstrates the agility of the system to targets. The number of failed simulations in the experiment is accumulated to reflect the robustness of the algorithm.

Comparison. To see whether IWS is valid or not, we compare it with the algorithm (named p-algorithm) with fixed wakening probability $\mathrm{p}$ evaluated in the interval $[0,1]$. While the parameter $\mathrm{C}$ in IWS is evaluated in the interval $[0,10]$.

In the simulation, the number of simulations is set to 30 . Let " $T$ " and " $E$ " be the mean value of 30 simulations respectively and " $F$ " be the times of failed simulations in the experiment. The results are as follows:

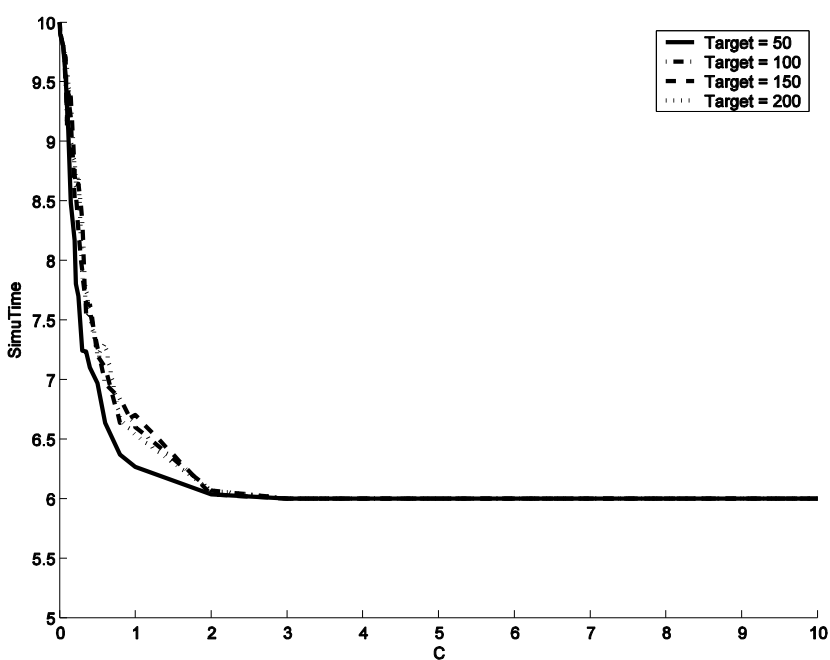

Figure 5. Simulation time with different target numbers of IWS

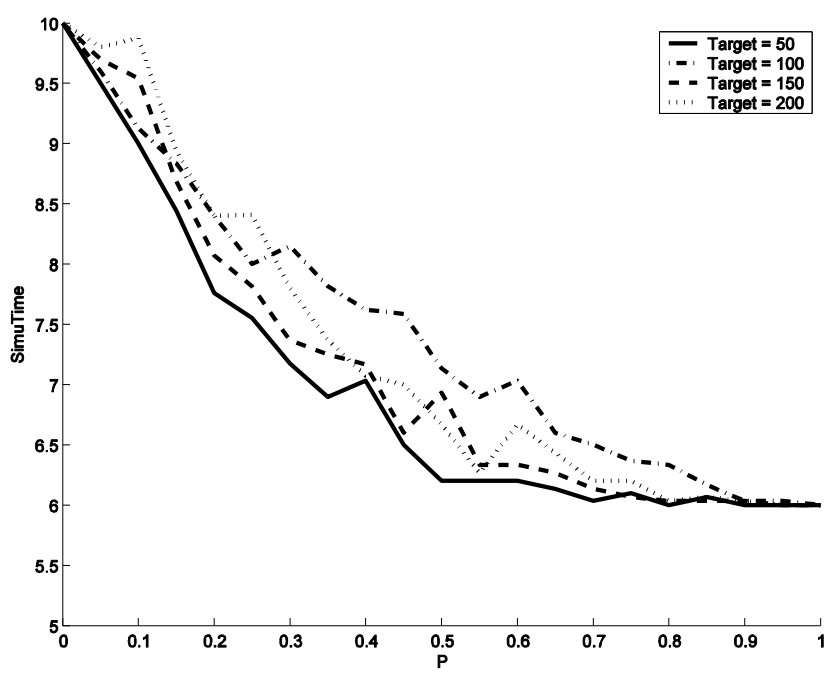

Figure 6. Simulation time with different target numbers of p-algorithm

Figure 5 and 6 show that simulation time is attenuating with the increase of parameter $C$ and probability p. When $C>2$ and $p>0.9$, simulation time remains stable at the value of 6 , which means the targets can be detected as soon as they are generated.

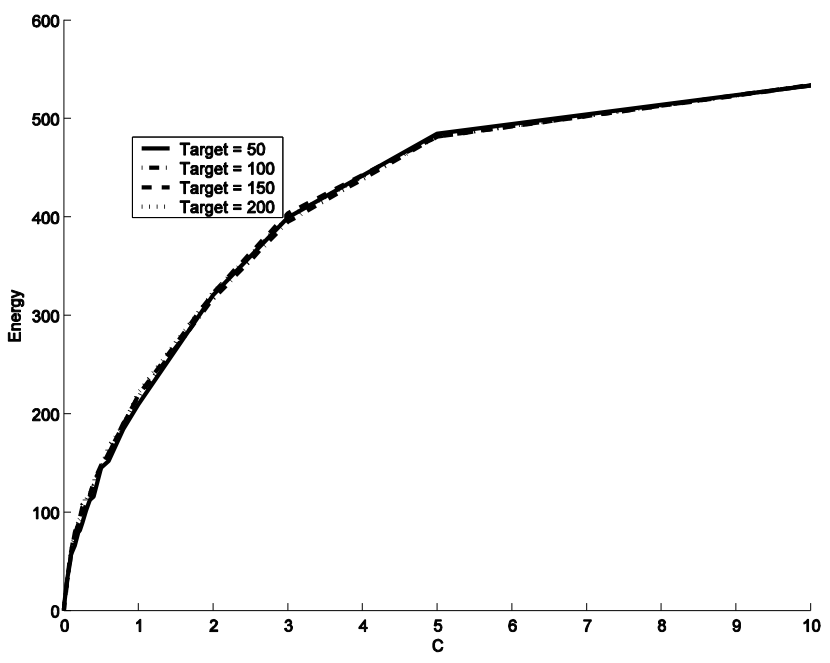

Figure 7. Energy cost with different target numbers of IWS 


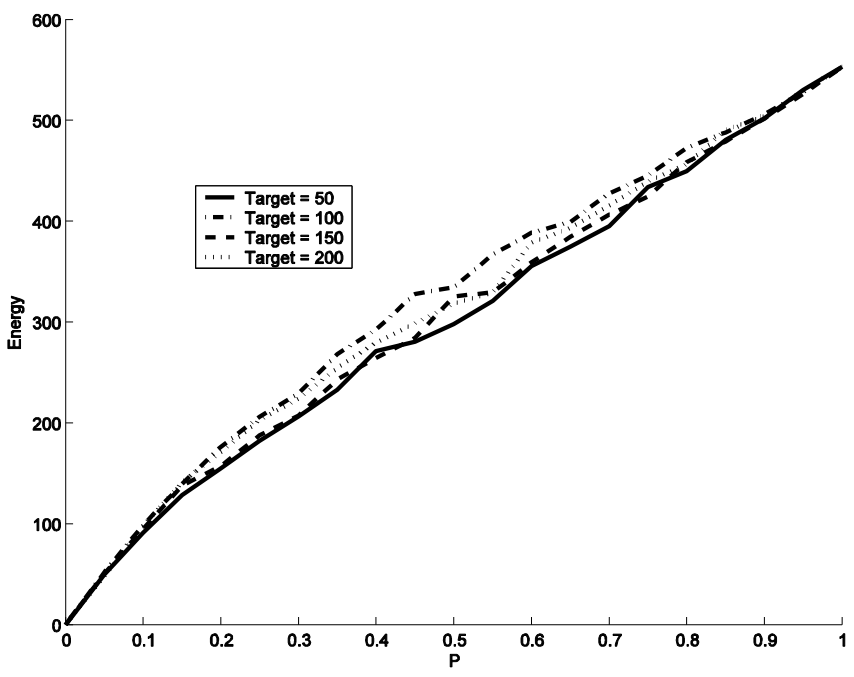

Figure 8. Energy cost with different target numbers of p-algorithm

Figure 7 and 8 illustrate that energy cost increases monotonously with parameter $C$ and probability $p$. Compared to p-algorithm, the energy cost of IWS is robust to different targets number $K$. From figure 5-8, we can also observe that $\mathrm{p}$ algorithm usually costs more energy than IWS with the same simulation time $T$.
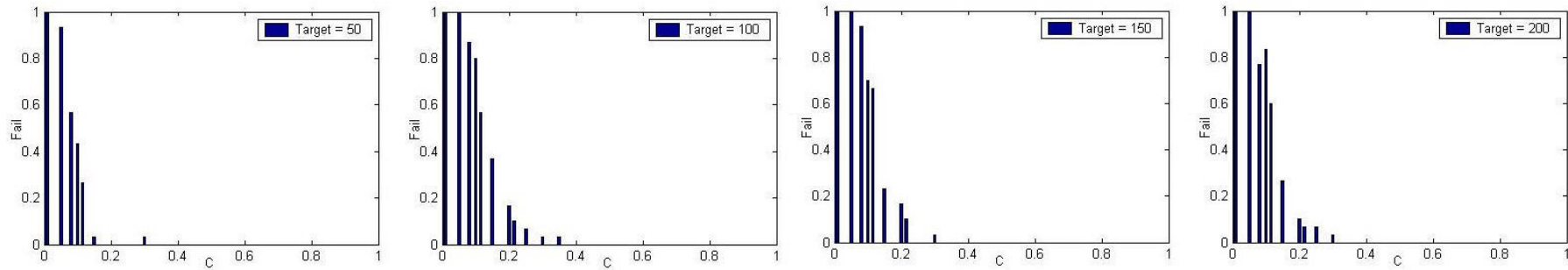

Figure 9. Failure time with different target numbers of IWS
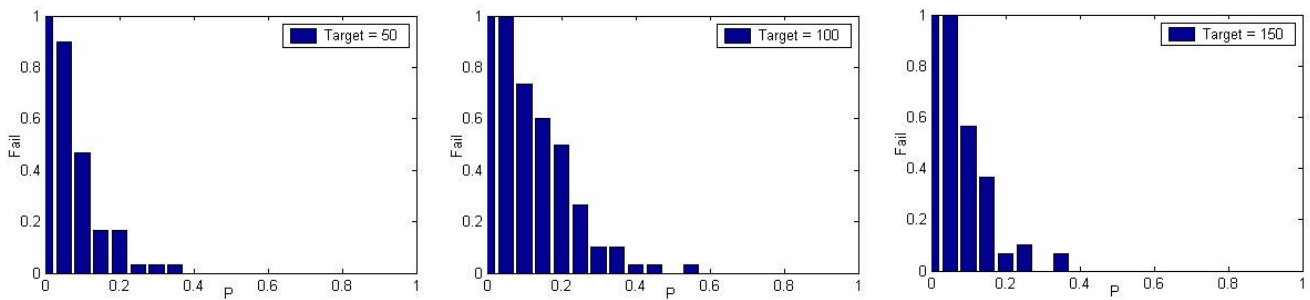

Figure 10. Failure time with different target numbers of p-algorithm
Figure 9 and 10 show the relationship of the failure times with parameter $C$ and $p$ when different targets numbers are applied. These figures indicate that failure time increases when either of parameter $C$ and $p$ decreases. Meanwhile, it's clear that with respect to the p-algorithm, failure times in IWS are less affected by the targets number $K$, which implies the

From the above simulation results we can draw the following conclusions:

- The energy cost $E$ is reduced by the decrease of $C$ and $p$. Besides, the failure times will increase if the parameter $C$ or $p$ decreases. $T$ and $E$ will increase through adding targets number $K$. Thereby, it is vital to reasonably choose the parameters $C$ according to different applications in order to ensure the optimizing performance of the system.

- IWS is superior to p-algorithm in performance. The main reason is that IWS not only calculates the geographical locations of the sensor nodes but also utilizes importance degree based scheduling scheme to raise efficiency. superior robustness of IWS.

\section{CONCLUSIONS}

We propose the IWS to save network resources through considering different importance degrees of blue-green algae surveillance locations. In the algorithm, importance degree is introduced to reflect the different importance of the grids in the surveillance zone. It is used to calculate the wakening probability which determines the modes of the sensor nodes. Our conducted green algae surveillance simulation results show that our approach is robust and energy efficient. Moreover, a compromise is achieved between energy cost and system agility. In the future, we will focus on the wake up mechanism based on comprehensive factor combination of region importance degree, sensor node energy and network connectivity.

\section{ACKNOWLEDGMENT}

This work was supported in part by the National Basic Research Program of China (973 Program) under Grant No.2011CB302803, National Natural Science Foundation of China under Grant No.61003292, Beijing Natural Science Foundation under Grant No.4092045 and the National S\&T Major Project of China under Grant No. 2010ZX03006-006. 


\section{REFERENCES}

[1] Dong Li, Ze Zhao, Li Cui, He Zhu, Le Zhang, Zhaoliang Zhang, and Yi Wang. The Design and Implementation of a Surveillance and SelfDriven Cleanup System for Blue-green Algae Bloom on Lake Tai. The 7th IEEE International Conference on Mobile Ad-hoc and Sensor Systems (MASS 2010), Demo Paper, pages 759-761, San Francisco, CA USA, Nov., 2010.

[2] S. Meguerdichian, F. Koushanfar, M. Potkonjak, and M.Srivastava. Coverage Problems in Wireless Ad-Hoc Sensor Network, Proc. IEEE INFOCOM '01, pp. 1380-1387, 2001.

[3] D. Tian and N. D. Georganas. A coverage-preserving node scheduling scheme for large wireless sensor networks. In First ACM International Workshop on Wireless Sensor Networks and Applications, pages 32-41, 2002.

[4] F. Ye, G. Zhong, S. Lu, and L. Zhang. PEAS: A Robust Energy Conserving Protocol for Long-lived Sensor Networks. In Proc. of International Conference on Distributed Computing Systems (ICDCS), May 2003.

[5] Liu, Changlei; Cao, Guohong; , "Spatial-Temporal Coverage Optimization in Wireless Sensor Networks," Mobile Computing, IEEE Transactions on , vol.10, no.4, pp.465-478, April 2011

[6] Dezun Dong; Yunhao Liu; Kebin Liu; Xiangke Liao; , "Distributed Coverage in Wireless Ad Hoc and Sensor Networks by Topological Graph Approaches," Distributed Computing Systems (ICDCS), 2010 IEEE 30th International Conference on , vol., no., pp.106-115, 21-25 June 2010

[7] Yang Xiao; Hui Chen; Kui Wu; Bo Sun; Ying Zhang; Xinyu Sun; Chong Liu; , "Coverage and Detection of a Randomized Scheduling Algorithm in Wireless Sensor Networks," Computers, IEEE Transactions on, vol.59, no.4, pp.507-521, April 2010

[8] H. P. Moravec, "Certainty grids for mobile robots," Technical Report Preprint, The Robotics Inst., Carnegie-Mellon Univ., 1986

[9] Viera M A M, et al. Scheduling nodes in wireless sensor networks: a Voronoi approach[A]. Local Computer Networks[C], 2003. LCN '03. Proceedings. 28th Annual IEEE International Conference on 20-24 Oct. 2003 Page(s):423 - 429

[10] Wei Ye, Heidemann, J, and Estrin, D, "An energy-efficient MAC protocol for wireless sensor networks," INFOCOM 2002. Twenty-First Annual Joint Conference of the IEEE Computer and Communications Societies. Proceedings. IEEE Vol 3, pp. 1567 - 1576 June 2002

[11] He T, Huang CD, Blum BM, Stankovic JA, Abdelzaher T. Range-Free localization schemes in large scale sensor networks, In: Proc. of the 9th Annual Int'1 Conf. on Mobile Computing and Networking. San Diego: ACM Press, 2003. 81-95.

[12] Adlakha, S.; Srivastava, M. Critical density thresholds for coverage in wireless sensor networks, Wireless Communications and Networking, 2003. WCNC 2003. 2003 IEEE, vol.3, no., pp.1615-1620 vol.3, 20-20 March 2003 